\title{
El costo de oportunidad de la mano de obra familiar en la economía de la producción lechera de Michoacán, México
}

\author{
The opportunity cost of family labor in \\ the economy of the dairy production \\ in Michoacan, Mexico
}

\section{O custo de oportunidade do trabalho familiar na economia da produção de leite em Michoacan, no México}

\author{
Randy Alexis Jiménez Jiménez ${ }^{1}$, Valentín Espinosa Ortiz² \& Diana Milena Soler Fonseca ${ }^{3}$ \\ ${ }^{1}$ Médico Veterinario Zootecnista, MSc en Ciencias de la Producción y la Salud Animal, Estudiante \\ Doctorado en Desarrollo Rural de la Universidad Autónoma Metropolitana de México. ${ }^{2}$ Médico Ve- \\ terinario Zootecnista, Especialista en Desarrollo Microrregional en Áreas de Asentamiento Humano, \\ MSc en Producción Animal, Doctor en Ciencias Agropecuarias y Recursos Naturales. ${ }^{3}$ Médico Vete- \\ rinario Zootecnista, Especialista en Nutrición Animal Sostenible, Magister en Desarrollo Rural \\ 1,2Departamento de Economía, Administración y Desarrollo Rural. Facultad de Medicina Veterinaria \\ y Zootecnia. Universidad Nacional Autónoma de México. Av. Universidad \#3000 Circuito Exterior, \\ Ciudad Universitaria. Coyoacán Código Postal 04510, México D.F. ${ }^{3}$ Escuela de Ciencias Agrícolas, \\ Pecuarias y del Medio Ambiente -ECAPMA. Universidad Nacional Abierta y a Distancia -UNAD.
}

Tunja. Boyacá. Colombia

1alexis.j2@gmail.com, 2veo@servidor.unam.mx, ${ }^{3}$ diana.soler@unad.edu.co

\section{Resumen}

El objetivo de esta investigación es analizar los factores asociados a la variación del margen de utilidad y la influencia del costo de oportunidad de la mano de obra familiar en las utilidades económicas de la lechería familiar del municipio de Maravatío, Michoacán. Se obtuvo información productiva y económica por medio de cuestionarios, entrevistas semiestructuradas y observación directa, utilizando la metodología de Investigación Acción Participativa. El costo de oportunidad de la mano de obra se estableció a partir de las opciones laborales de los miembros familiares que participan en la actividad; las opciones se clasificaron en tres tipos: local, regional y extranjero, de acuerdo a la localización de las oportunidades laborales. Para determinar la influencia de la mano de obra familiar en las ganancias se empleó un modelo de regresión múltiple con inclusión de variables por pasos. El alimento del ganado representó el mayor índice de determinación de la variación del margen de utilidad con el $68 \%$. La mano de obra familiar es una de las variables que influye negativamente en la rentabilidad de las unidades de producción, representó $26 \%$ de la variación de la ganancia económica, con una relación lineal negativa de -1.05 ( $\mathrm{P} \leq 0.001)$. Las mejores alternativas laborales de los productores al emigrar manifiestan que la mano de obra familiar no es el factor productivo que les permite tener ganancias; sin embargo, la actividad lechera provee sustento productivo y económico en personas con un costo de oportunidad local y regional.

Palabras clave: mano de obra familiar, costo de oportunidad, ganancia económica, lechería familiar. 


\section{Abstract}

The objective of this research is to analyze associated factors with the variation of the profit margin and the influence of the opportunity cost of family labor in the economic profit of the family dairy in the municipality of Maravatio, Michoacan. Productive economic information was obtained through the use of questionnaires, semi-structured interviews and direct observation, using the methodology of Participatory Action Research. The opportunity cost of workforce was established on the basis of the work options for the family members involved in the activity; the options were classified into three types: local, regional, and foreign, according to the location of the job opportunities. In order to determine the influence of family labor in earning, it was used a multiple regression model with inclusion of step-by-step variables. The livestock feed represented the highest index of determination of the profit margin variation with the 68 $\%$. Family labor is one of the variables that have an adverse impact on the profitability of the production units, it represented $26 \%$ of the variation of economic gain, with a negative linear relation of -1.05 ( $P \leq 0.001)$. The best labor alternatives for producers, when emigrating, show that family labor is not the productive factor allowing them to have earnings; however, the dairy activity provides productive and economic sustenance for people with a local and regional opportunity cost.

Key-words: family labor, opportunity cost, economic gain, family dairy.

\section{Resumo}

O objetivo deste estudo é analisar os fatores associados com a variação das margens de lucro ea influência do custo de oportunidade da mão de obra familiar nos lucros econômicos da leiteria familiar do Municipio Maravatío, Michoacán. Obtevese informação produtiva e econômica por meio de questionários, entrevistas semi-estruturadas e observação direta, usando a metodologia de Pesquisa Ação Participativa. O custo de oportunidade de mão de obra foi estabelecido a partir das opções de trabalho dos membros da família que participam da atividade; opções foram classificadas em três tipos: locais, regionais e estrangeiros, de acordo com a localização de oportunidades de emprego. Para determinar a influência da renda da mão de obra familiar no ganho foi usada uma regressão com a inclusão progressiva de variáveis. Alimentação do gado representou o maior índice de determinação da variabilidade da margem de lucro com $68 \%$. O trabalho familiar é uma das variáveis que afetam negativamente a rentabilidade das unidades de produção, responsável por $26 \%$ da variação do lucro econômico, com uma relação linear negativa de $-1,05$ ( $p \leq 0.001)$. A melhor alternativa de trabalho para os produtores emigrantes, afirma que o trabalho familiar não é fator produtivo que lhes permite obter lucros; no entanto, a atividade leiteira fornece suporte produtivo e econômico para as pessoas com um custo de oportunidade local e regional.

Palavras-chave:mão de obra familiar, custo de oportunidade, lucro econômico, leiteria familiar.

\section{Introducción}

La producción de leche en México se caracteriza por su heterogeneidad tanto en la producción como en la comercialización de sus productos. Se identifican cuatro sistemas de producción: especializado, semiespecializado, doble propósito y familiar (Gallardo, 2004). Este último es el de menor tecnificación, sus hatos oscilan entre 2 y 10 vacas con rendimientos de 6 a 12 litros por vaca, este sistema tiene $10 \%$ de participación en la oferta nacional de leche (SAGARPA, 2004) y representa el 77\% del total de las unidades de producción (Martínez \& Salas, 2002).

A partir de la globalización se han observado transformaciones en el escenario mundial, una de ellas ha sido la disminución en el número de unidades (García et al., 2005). Esta tendencia se ha observado en Estados Unidos, Canadá, y algunos 
países europeos. En EU se señala que el número de granjas disminuyó entre 1992-2000 en 48,510 granjas, lo que significa una pérdida del $36.9 \%$; en Canadá en 1970 el número de unidades correspondía a 143,315, a principios del reciente siglo el número de granjas es de 21,911, es decir actualmente existen tan sólo el 7\% de las que existían en 1970; en países europeos como Bélgica se ha observado que el número de granjas ha disminuido hasta en un $69 \%$ en los últimos 10 años (Schwarzweller \& Davidson, 2000). En cada país existen motivos diferentes que explican la disminución, tales como: problemas en la tenencia de la tierra, políticas de fomento hacia el gran productor, situaciones de inseguridad, entre otros (García et al., 2005).

En México la tendencia de decrecimiento de la pequeña producción ha sido poco estudiada, se ha documentado que ha disminuido su participación en la oferta nacional de leche del 21 al $10 \%$ de 1980 al 2000. Esta disminución se ha relacionado con altos costos de producción, baja calidad de la leche, menor tecnificación (García et al., 2005), y poca integración a las cadenas productivas, disminuyendo sus ingresos por la intermediación (Espinosa et al., 2002). Adicionalmente, la importación de leche a bajos precios ha disminuido la competitividad de estas unidades de producción (Espinoza et al., 2005), provocando la baja rentabilidad y la consecuente salida del mercado.

Se menciona que las unidades de producción familiar tienen ventajas en el ámbito socioeconómico, debido a que mejoran la alimentación familiar (Hoorwerg et al., 2000), muestran sustentabilidad ecológica (Brunett, 2004; Espinosa, Wiggins, González \& Aguilar, 2004), se obtienen ingresos a partir de la venta de leche y generan empleo. Es por eso que la actividad lechera en el medio familiar es considerada una opción de desarrollo (Arriaga et al., 1999; Wiggins et al., 2001).

Se considera que una de las limitantes de este sistema para lograr competitividad económica es la falta de visión empresarial, y por ello, no es común que consideren dentro de sus actividades el registro productivo y económico (SAGARPA, 2004). Adicionalmente, esa falta de visión conlleva a que insumos tan relevantes como la mano de obra familiar no sean considerados como factor económico dentro de sus costos.

Por lo anterior los métodos empleados para la evaluación y análisis económico de estas unidades han propuesto valorar el uso de la mano de obra familiar, con un costo de oportunidad (Wiggins et al., 2001; Jiménez et al., 2005). Este costo se define como el valor del insumo en el mejor uso alternativo (Leftwitch, 1987; Alonso et al., 1989; Alonso, 2007).

En los estudios que analizan económicamente a la actividad contabilizando la mano de obra familiar, se ha observado que a pesar de la diversidad en el margen de utilidad donde unas unidades de producción mantienen ganancias (Espinoza et al., 2005), y otras pérdidas (Jiménez et al., 2005), el costo de oportunidad sugiere una ganancia o un sueldo implícito al productor, respectivamente. Sin embargo, con la heterogeneidad que caracteriza a este sistema de producción no se han analizado los factores asociados a la variabilidad en el margen de utilidad, y en especial, la magnitud que representa el costo de oportunidad de la mano de obra familiar asociada a la obtención de ganancias o pérdidas en la producción de leche del sistema familiar. Por tales razones, el presente estudio tiene como propósito analizar los factores asociados a la variación del margen de utilidad y la influencia del costo de oportunidad de la mano de obra familiar en las ganancias o pérdidas de la lechería de producción familiar de Maravatío, Michoacán.

\section{Metodología}

El estudio se realizó en 16 unidades de producción familiar en el municipio de Maravatío, Michoacán, México, elegidas por su disponibilidad para trabajar en el estudio y por poseer características que identifican al sistema de producción familiar, como es el tamaño del hato: el $70 \%$ de los productores 
poseen de 2 a 10 vacas, el resto 10 a 20 vacas, y el predominio de recursos originados en la unidad familiar como es: la mano de obra, las instalaciones, la recría, entre otros.

El municipio se localiza en la región centro occidente del país en las coordenadas 1954' de latitud norte y 10027' de longitud oeste, a una altitud de 2,020 metros sobre el nivel del mar. Su clima es templado con temperaturas que oscilan entre $14.1^{\circ} \mathrm{C}$ y $29.9^{\circ} \mathrm{C}$, y lluvias en verano con una precipitación pluvial anual de 897.7 milímetros.

La elección del área de estudio se definió por dos aspectos que distinguen al estado de Michoacán y al municipio de Maravatío. Por un lado, el estado de Michoacán se considera abastecedor de leche y productos lácteos a la Ciudad de México (Losada et al., 2001), mientras que Maravatío, por su localización geográfica se considera centro interconexión a grandes centros urbanos como son Morelia y Toluca, capitales de los estados de Michoacán y México, respectivamente. Por otro lado, Michoacán se caracteriza por producir predominantemente bajo los sistemas de producción semiespecializado y familiar (SAGARPA, 2004), es así que en Maravatío predominan unidades de producción de tipo familiar.

Mediante la metodología de Investigación Acción Participartiva (De Schutter, 1989), se obtuvo información productiva y económica mediante cuestionarios, entrevistas semiestructuradas y observación directa, realizando 6 visitas por unidad $(n=96)$. Se siguió esta metodología ya que se optimiza la relación de confianza entre investigador e investigado, aspecto sumamente importante para obtener datos económicos.

Para determinar la contribución económica de la mano de obra familiar en las ganancias, se establecieron como variable de respuesta las ganancias o pérdidas obtenidas en la producción y como variables explicativas el costo de los principales insumos en la producción (mano de obra familiar, alimento, mano de obra asalariada y agotamiento animal), el precio de venta, las horas totales de mano de obra familiar y el mes de estudio. Cabe señalar que estas dos últimas variables al incluirlas en el modelo estadístico no contribuyeron a la variación de la ganancia económica.

Los costos de producción se calcularon por el método por insumos, utilizado en el Departamento de Economía, Administración y Desarrollo Rural de la FMVZ de la UNAM (Alonso et al., 1989). Los precios de los insumos y los costos obtenidos se presentan en dólares (US\$).

El costo de oportunidad de la mano de obra familiar se consideró como costo fijo, debido a que su valor no se modifica si aumenta o disminuye la producción en el corto plazo. El costo de oportunidad de la mano de obra familiar se estableció a partir de las alternativas laborales que pudieran acceder de manera local (jornal en la zona), regional (salario mínimo de la zona) y extranjeras (dólares por hora), de acuerdo a las expectativas de empleo que los productores y familiares que laboran en la actividad mencionaron.

Para determinar la influencia de las variables explicativas en las utilidades unitarias se utilizó un modelo de regresión lineal múltiple. Se empleó el método de selección de variables por pasos (Stepwise), con un criterio de inclusión de las variables de $\mathrm{P}=0.1$, bajo dos perspectivas: una de ellas consideró el costo de oportunidad de la mano de obra familiar y la otra no lo consideró. La suma de cuadrados parcial atribuida a cada variable se usó para determinar la magnitud en que contribuyó a la variación de la ganancia económica.

\section{Resultados}

Los costos de producción promedio por litro de leche fueron de US $\$ 0.61$ considerando el costo de oportunidad de la mano de obra familiar, y sin considerarlo de US\$0.36 (Tabla 1), estos costos son superiores al precio de venta medio de US\$0.30, por lo que en promedio las unidades de producción presentaron pérdidas. 
Tabla 1. Costos unitarios promedio y su estructura en el costo total

\begin{tabular}{lllcc}
\hline \multicolumn{1}{c}{ Concepto } & Promedio (US\$) & SD $^{1}$ & \% con MOF & \% sin MOF \\
\hline Costos Fijos & & & & \\
\hline Mano de obra familiar & & & \\
\hline Agotamiento animal & 0.24 & 0.26 & 37.37 & 9.73 \\
\hline Equipo & 0.03 & 0.03 & 5.44 & 4.97 \\
\hline Instalaciones & 0.02 & 0.03 & 3.13 & 3.76 \\
\hline Otros costos fijos & 0.01 & 0.02 & 2.16 & 3.36 \\
\hline Subtotal & 0.01 & 0.01 & 1.80 & 21.83 \\
\hline Costos Variables & 0.31 & 0.28 & 49.90 & \\
\hline Alimento & & & & 55.58 \\
\hline Mano de obra asalariada & 0.21 & 0.23 & 34.70 & 8.75 \\
\hline Fármacos y biológicos & 0.04 & 0.11 & 7.40 & 6.40 \\
\hline Otros costos variables & 0.02 & 0.03 & 4.04 & 7.45 \\
\hline Subtotal & 0.02 & 0.02 & 3.96 & 78.18 \\
\hline Costo Total Unitario con MOF & & 0.31 & 50.10 & \\
\hline Costo Total Unitario sin MOF & & & \\
\hline
\end{tabular}

'Desviación estándar, ${ }^{2}$ Mano de obra familiar, ${ }^{3}$ Promedio ponderado

Asimismo, el costo de oportunidad de la mano de obra familiar en el modelo de regresión fue el segundo insumo que explicó la variación de la ganancia económica cuando se considera este insumo (Tabla 2), por lo que la mano de obra no es la principal, ni la única variable que determina la variación de la ganancia económica.
Las variables de impacto económico para la variación de la ganancia económica al considerar el costo de oportunidad de la mano de obra familiar fueron los costos unitarios de alimento con un $68.29 \%$, mano de obra familiar con el $26.62 \%$, mano de obra asalariada con un $3.75 \%$, agotamiento animal con un $0.55 \%$ y precio de venta con el $0.20 \%$ ( $\mathrm{P} \leq 0.001)$, con un coeficiente de determinación $\left(R^{2}\right)$ de 0.99 (Tabla 2).

Tabla 2. Variables ${ }^{1}$ que contribuyen económicamente a la variación de la ganancia considerando el costo de oportunidad de la mano de obra familiar

\begin{tabular}{lcccc}
\hline \multicolumn{1}{c}{ Variable } & $\begin{array}{c}\text { Coeficiente } \\
\text { de regresión }\end{array}$ & $\begin{array}{c}\text { Error } \\
\text { estándar }\end{array}$ & $\begin{array}{c}\boldsymbol{R}^{2} \\
\text { parcial }\end{array}$ & $\begin{array}{c}\boldsymbol{R}^{2} \\
\text { acumulado }\end{array}$ \\
Alimento & -0.11 & 0.002 & 0.6829 & 0.6829 \\
$\mathrm{MO}^{2}$ familiar & -0.10 & 0.001 & 0.2662 & 0.9491 \\
$\mathrm{MO}^{2}$ asalariada & -0.09 & 0.004 & 0.0375 & 0.9866 \\
Agotamiento animal & -0.16 & 0.024 & 0.0055 & 0.9921 \\
Precio de Venta & 0.07 & 0.013 & 0.0020 & 0.9941 \\
\hline
\end{tabular}

${ }^{1} \mathrm{P} \leq 0.001 .{ }^{2}$ Mano de obra 
Las variables determinantes de la variación en la ganancia sin considerar el costo de oportunidad ( $\mathrm{P} \leq 0.001)$ fueron, los costos unitarios de alimento con el $90.91 \%$, mano de obra asalariada con el 5.75\%, agotamiento animal con el $1.81 \%$ y el precio de venta con el $0.4 \%$, con un $R^{2}$ de 0.98 , (Tabla 3 ).

Tabla 3. Variables ${ }^{1}$ que contribuyen económicamente a la variación de la ganancia sin considerar el costo de oportunidad de la mano de obra familiar.

\begin{tabular}{lcccc}
\hline \multicolumn{1}{c}{ Variable } & $\begin{array}{c}\text { Coeficiente } \\
\text { de regresión }\end{array}$ & Error estándar & $\boldsymbol{R}^{2}$ parcial & $\boldsymbol{R}^{2}$ acumulado \\
\hline Alimento & -0.10 & 0.002 & 0.9091 & 0.9091 \\
\hline $\mathrm{MO}^{2}$ Asalariada & -0.09 & 0.004 & 0.0575 & 0.9666 \\
\hline Agotamiento Animal & -0.19 & 0.022 & 0.0181 & 0.9847 \\
Precio de Venta & 0.07 & 0.013 & 0.0040 & 0.9887 \\
\hline
\end{tabular}

${ }^{1} \mathrm{P} \leq 0.001,{ }^{2}$ Mano de obra

Las variables mes de estudio y horas totales de mano de obra familiar, no contribuyeron significativamente $(P \geq 0.05)$ a la variación de la ganancia económica en su selección para el modelo de regresión múltiple en los análisis desarrollados.

Cabe señalar que debido a la disparidad que se observa en la distribución del número de familiares respecto al tipo de costo de oportunidad (Tabla 4), se utilizó un costo de oportunidad ponderado tanto en el cálculo del costo de producción, como en el modelo de regresión lineal múltiple.

En las 16 unidades de producción evaluadas se encontró una participación de 2 familiares por unidad, en promedio, los cuales dedican nueve horas a la actividad lechera. La mayoría de los familiares presentó un costo de oportunidad local; este grupo se distingue por ser personas sin posibilidades de emigrar o de encontrar empleo en otra actividad, son mujeres que se han quedado a cargo de las unidades de producción y niños que las apoyan. Las personas con un costo de oportunidad regional son mujeres con escolaridad superior a secundaria, las cuales con oportunidades de empleo en la región y con salarios mínimos, apoyan a sus familias en la actividad lechera. Los familiares sin costo de oportunidad son personas de mayor edad (Tabla 4), lo que impide que tengan una alternativa de empleo remunerado. Bajo estos esquemas de oportunidad, este tipo de personas utilizan la producción de leche para generar su empleo y complementar sus ingresos, con la satisfacción de permanecer en su comunidad y con sus familias.

Tabla 4. Análisis del costo de oportunidad de la mano de obra familiar en el periodo de estudio.

\begin{tabular}{lcccccc}
\hline \multicolumn{1}{c}{ Tipo de CO } & Personas & Edad & SD $^{2}$ & CO $^{1 / h o r a}$ & SD $^{2}$ & COP $^{3} /$ hora $^{\prime}$ \\
Local & 104 & 31 & 14.53 & $\$ 1.61$ & $\$ 0.93$ & $\$ 1.22$ \\
Regional & 8 & 32 & 3.20 & $\$ 1.42$ & $\$ 0.00$ & $\$ 0.08$ \\
Extranjero & 26 & 38 & 6.44 & $\$ 14.01$ & $\$ 3.61$ & $\$ 2.64$ \\
Sin CO & 42 & 56 & 10.63 & $\$ 0.00$ & - & $\$ 0.00$ \\
\hline
\end{tabular}

${ }^{1}$ Costo de oportunidad, ${ }^{2}$ Desviación estándar, ${ }^{3}$ Costo de oportunidad ponderado 
Por otra parte, la mano de obra familiar que tiene un costo de oportunidad extranjero son principalmente jefes de familia y adultos jóvenes en edad laboral, se caracterizan por el constante flujo entre el mercado laboral estadounidense y su comunidad. Durante su permanencia temporal se dedican a la actividad lechera, con lo cual apoyan a sus familias, permitiéndoles tener un ingreso y ocupación durante su estadía en la comunidad.

La percepción de las oportunidades de empleo de familiares que laboran en la actividad lechera va muy de la mano con la edad, con la escolaridad y con el sexo; a mayor edad y sin escolaridad, las oportunidades son muy bajas; a una edad laboral con o sin escolaridad y de sexo masculino su oportunidad es mayor tanto en el mercado local como en el extranjero; mientras que familiares del sexo femenino con escolaridad y en edad laboral su oportunidad es intermedia en el mercado regional y local. Por lo que pueden existir variables sociales que se reflejan en las oportunidades de empleo y en la economía de las unidades de producción familiar.

\section{Discusión y conclusiones}

De acuerdo a los resultados obtenidos, se observa que la variación en la ganancia no está determinada únicamente por el costo de oportunidad de la mano de obra familiar, además de este insumo, los costos por alimento, el agotamiento animal, la mano de obra asalariada y el precio de venta son las variables que influyen de manera directa en la variación de las utilidades generadas. El costo por los insumos alimenticios es la variable que tiene el mayor efecto en esta variación cuando se considera el costo de oportunidad de la mano de obra familiar (68\%), inclusive su efecto se incrementa cuando no se considera el costo de oportunidad (90\%), mientras que las otras variables se mantienen constantes.

El coeficiente de determinación que manifiesta el costo de los insumos alimenticios del ganado en la variación de la ganancia, muestra la naturaleza variable de este insumo. Esta variabilidad la origina la naturaleza de los insumos, ya que muchas veces al ser obtenidos a través de la agricultura la calidad y precios varían en el corto plazo (Alonso et al., 1989, Arriaga et al., 1999). En los sistemas de producción familiar, esta diversidad se magnifica por los cambios en las dietas que los productores ofrecen al ganado en las diversas temporadas del año. En Maravatío, Jiménez-Jiménez et al. (2011) mencionan que la alimentación del ganado depende de la época del año, predominando forrajes secos y la compra de insumos en los meses de octubre a abril, y el uso de forrajes húmedos y pastoreo de praderas mixtas en los meses de mayo a septiembre.

El alimento en la gran mayoría de las actividades pecuarias y en particular en la producción de leche es el insumo de mayor peso en los costos, (Arriaga et al., 1999; Espinoza et al., 2005). Su relación con las ganancias es negativa, en este estudio se estimó una disminución promedio de US\$0.10 en el costo de este insumo por unidad de ganancia (Tablas 2 y 3). Sin embargo, en la lechería familiar al estar ligada tradicionalmente a la actividad agrícola (Martínez \& Salas, 2002), se procura su autosuficiencia con recursos propios, que en algunos casos, el productor no dimensiona el costo de estos insumos.

En algunas investigaciones participativas, por la importancia económica que tiene este insumo, se han generado y evaluado estrategias productivas y alimentarias (Arriaga-Jordán, 2002; Albarrán-Portillo, Espinosa-Ortega, García-Martínez \& Castelán-Ortega, 2002; Espinoza et al., 2005) que permiten tener una mayor producción de leche y disminuir los gastos por alimentos comerciales, con el fin de disminuir la dependencia de insumos externos. Este tipo de prácticas también son muy notorias en unidades de producción de Maravatío, ya que incorporan ingredientes de su propia producción en la dieta, como maíz, esquilmos agrícolas, alfalfa, trébol y avena (Jiménez-Jiménez, Espinosa, Alonso, García, Dávalos \& Gil, 2011), con lo cual sobrellevan los gastos y permiten que la actividad les retorne beneficios económicos. 
En relación al costo de oportunidad de la mano de obra familiar, es evidente que al considerarlo en la contabilidad de costos, influya negativamente en la rentabilidad de estas unidades de producción. El valor que alcanza por el costo de oportunidad extranjero hace que se localice como el principal insumo en la estructura de los costos y en el modelo de regresión es la segunda variable que contribuye a la variación de la ganancia. La magnitud del valor otorgado a la mano de obra familiar en el modelo de regresión múltiple permite estimar una disminución promedio de US\$0.10 en el costo de este insumo por unidad de ganancia. Lo anterior evidencia la relevancia económica de este insumo en las unidades de producción familiar, por lo que se vuelve fundamental el considerarlo en la evaluación económica, para mejorar su participación y hacer más eficiente su uso.

En otras evaluaciones económicas del sistema de producción familiar (Arriaga et al., 1999; Wiggins et al., 2001; Espinoza, Álvarez, Del Valle \& Chauvete, 2005), se ha considerado a la mano de obra familiar como un costo variable. Estos autores mencionan que esta inclinación a lo variable, radica en que los familiares que laboran en la actividad en cualquier momento pueden abandonarla o cambiar de preferencia en la diversidad de actividades que tienen dentro de la unidad familiar, restándole importancia a la producción de leche. No obstante, como se indicó en la metodología, al ser una actividad de ocupación familiar la participación de los miembros no se modifica si aumenta o disminuye la producción en el corto plazo, por lo que es posible considerar a este insumo como fijo. Debido al modelo estadístico utilizado y el análisis realizado es posible abonar a esta última afirmación, dado que el coeficiente de determinación del costo de oportunidad de mano de obra familiar representa el $27 \%$, y no es tan determinante en la variación de la ganancia con respecto a la alimentación, y es más notorio cuando no se considera la mano de obra familiar en modelo de regresión múltiple. Por lo cual, el análisis ayuda a sostener la naturaleza fija del costo de oportunidad de la mano de obra familiar en el corto plazo.
Las desventajas de utilizar un costo de oportunidad para la mano de obra familiar, radican en subestimar o sobrestimar las mejores alternativas, o que no exista tal (Kay, 1986). Incluirlo en el análisis económico de las unidades familiares aun cuando tienen una lógica diferente de producción, se da en razón a que están vinculados al mercado de insumos y productos, permitiendo evaluar en conjunto todos los factores de producción. En la presente investigación al incorporarlo, también muestra su impacto en la generación de beneficios y deterioros socioeconómicos que ocurren en la lechería familiar.

En las otras variables que influyen en la variación de la ganancia, como el caso de la mano de obra asalariada, su contribución es reflejo de los efectos que ocasiona la migración (Nava-Tablada \& Marroni, 2003), su uso se ha incrementado en la actividad. En 8 de las 16 unidades evaluadas, en las cuales existe un uso regular de este insumo, se observó el fenómeno migratorio de alguno de los familiares. Sin embargo, la migración de personas en edad laboral no es la única causa que motiva a contratar personal, también se incrementa en unidades con mayor número de vacas (Arriaga et al., 1999), en el estudio se evidenció en hatos de más de 10 vacas. Adicionalmente, productores en edad avanzada y productores que aprovechan el pastoreo de praderas comunales en época de lluvias contratan personal para colaborar en estas actividades. Principalmente, niños y jóvenes constituyen la mano de obra asalariada que favorece la persistencia de estas unidades de producción, y su uso debido al envejecimiento de la población y a la migración podría ir en aumento.

Analizando el efecto el agotamiento animal, a pesar de que su influencia en la variación de ganancia es mínima, su relación con esta última representa el mayor coeficiente estimado, se observó una disminución promedio de US\$0.18 en el costo de este insumo por unidad de ganancia. La baja contribución en la variación de las ganancias es resultado de la vida útil de las vacas, pues se puede extender a 
más de 8 años en el sistema de producción familiar, a diferencia de los sistemas especializados donde el promedio es de 3 años (Valdespino, 1993). Este periodo productivo de las vacas y la recría que ellos mismos realizan, da pauta para que este insumo no tenga una mayor representación en la economía de las unidades de producción. Por otro lado, el alto coeficiente estimado es reflejo de la productividad de los animales. El rendimiento medio encontrado fue de 11 litros por vaca, hasta con $50 \%$ de vacas secas en los hatos, esto refleja que a medida que los productores aumenten la producción por vaca y se disminuya el número de vacas secas, el costo de producción tenderá a disminuir y por ende incrementará sus ganancias.

Por último, el precio de venta de la leche a pesar de ser un factor que influye directamente en el margen de utilidad de cualquier empresa, fue la variable con menor influencia en la variación de las ganancias. Sin embargo, el precio de venta es relevante a la hora de la toma de decisiones, pues el aumento en el precio puede repercutir en los incrementos en la producción (Espinoza et al., 2005), en el tamaño del hato y en la mano de obra destinada a la actividad (Zepeda \& Jongsoog, 2006). Cabe señalar que el precio de venta de la leche no depende directamente del productor, ya que puede estar determinado por el intermediario, por las variaciones estacionales de la producción (Méndez, Tzinzun \& Val, 2000), por la calidad del producto (McDonald, 1999) y por las imperfecciones del mercado. Por lo anterior el precio de venta es una variable económica que determina el manejo de los factores de la producción y se refleja en el ingreso del productor.

En conclusión, la variabilidad en la obtención de ganancias en la producción de leche de Maravatío Michoacán está influenciada por la mano de obra familiar y asalariada, el agotamiento animal y el precio de venta, pero particularmente por la alimentación del ganado. La generación de ganancias no sólo reside en la mano de obra familiar, sino también en la disminución de los costos de los principales insumos, en el uso eficiente de los recursos y en la búsqueda de mejores precios de venta de la leche.

El uso de la mano de obra familiar en las unidades de producción evaluadas no es el insumo que les permite tener ganancias. Su valoración con un costo de oportunidad demostró que contribuye de manera negativa en la generación de ganancias, ya que en la comunidad se poseen oportunidades laborales de alto valor cuando se emigra.

El estudio evidenció la relevancia económica que tiene la mano de obra familiar en la producción familiar, por lo que se vuelve fundamental valorar la mano de obra familiar de manera explícita en la contabilidad de costos y gastos que los productores realizan, con el fin de mejorar su participación y hacer más eficiente su uso.

La migración presente en regiones como Maravatío, además de implicar un alto costo de oportunidad para la mano de obra familiar, ha implicado también una reestructuración laboral, donde el aumento en la participación de mujeres, niños y ancianos, así como el uso de mano de obra asalariada, permite la permanencia de la actividad lechera en la comunidad.

La mano de obra familiar también tiene repercusiones económicas positivas en la actividad lechera de la comunidad, pues genera ingresos al volverse factor productivo en personas con pocas expectativas de trabajo y de adultos mayores y también genera un bien, que satisface demandas locales.

\section{Agradecimientos}

Se agradece al Programa de Apoyo a Proyectos de Investigación e Innovación Tecnológica PAPIIT de la UNAM (proyecto IN308613-NR308613) por el financiamiento para realizar el trabajo y a los productores del Maravatío, Michoacán, México por facilitar información. 


\section{Literatura citada}

1. Alonso, F. A., Bächtold, E., Aguilar, A., Juárez, J., Casas, V. M., Meléndez J. R, Huerta, E., Mendoza, E. \& Espinoza, A. (1989). Economía Zootécnica. Segunda Edición. Noriega Editores. México. 751 p.

2. Alonso, F. A. (2007). Costos de producción. En: Economía Agropecuaria. México D.F.: Grupo Vanchri.

3. Arriaga, C. M., Espinoza, A., Rojo, H., Valdés, J. L., Sánchez, E. \& Wiggins, S. (1999). Aspectos Socio-económicos de la producción campesina de leche en el Valle de Toluca: I. Evaluación económica inicial. Agrociencia 33 (4): 483-491. México.

4. Arriaga-Jordán, C. M., Albarrán-Portillo, B., EspinozaOrtega, A., García-Martínez, A. \& Castelán-Ortega, O. A (2002). On-farm comparison of feeding strategies based on forages for small-scale dairy production systems in the highlands of central Mexico. Expl Agric 38 (4): 375-388. Cambridge University Press, United Kingdom

5. Brunett, L. (2004). Contribución a la evaluación de sustentabilidad, estudio de caso de 2 agroecosistemas campesinos de maíz y leche del Valle de Toluca. Doctorado en ciencias de la salud y producción animal. Facultad de Medicina Veterinaria y Zootecnia, UNAM, México D.F. 188 p.

6. De Schutter A. (1989). Investigación Participativa: una opción metodológica para la educación de adultos. Retablo de papel 3. 4ㄹ ed. Organización de Estados Americanos (OEA), CREFAL, Michoacán, México. 392 p. México

7. Espinosa, V., López, C. A., García, G., Gómez, L., Velásquez, P. \& Rivera G. (2002). Márgenes de comercialización de la lecha cruda producida en sistema familiar. Revista Científica 12 (Suplemento 2), Octubre: 650-654. Venezuela

8. Espinosa, J. A., Wiggins, S., Gonzáles, A. T. \& Aguilar, U. (2004). Sustentabilidad económica a nivel de empresa: aplicación a unidades familiares de producción de leche en México. Téc Pec Méx 42 (1): 55-70. México

9. Espinoza, A., Álvarez, A., Del Valle, C. \& Chauvete, M. (2005). La economía de los sistemas campesinos de producción de leche en el altiplano mexicano. Téc pec Méx. 43 (1): 39-56. México

10. Gallardo, J. (2004). Situación actual de la leche de bovino en México. Revista claridades agropecuarias. ASERCASAGARPA. 136: 3-32. México

11. García, L. A., Aguilar, A., Luévano A. \& Cabral, A. (2005). La globalización productiva y comercial de la leche y sus derivados. Articulación de la ganadería intensiva lechera de la comarca lagunera. Plaza y Valdés editores, Universidad Autónoma Metropolitana Unidad Xochimilco, México. 278 p.

12. Hoorweg, J., Leegwater, P. \& Veerman, W. (2000). Nutrition in agricultural development: Intensive dairy farming by rural smallholders. Ecology of Food and Nutrion 39 (6): 395-416.

Recibido: 4 de septiembre de 2013

Aceptado: 17 de octubre de 2013
13. Jiménez, R. A., Espinosa V., García, G., Alonso A., Meléndez, R. \& García, L. A. (2005). Utilidad económica de la lechería familiar considerando el costo de oportunidad de la mano de obra. In: Memorias de la XLI Reunión Nacional de Investigación Pecuaria. Cuernavaca, Morelos, México. pp: 289.

14. Jiménez-Jiménez, R. A., Espinosa, O. V., Alonso, P. F. A., García, H. L. A., Dávalos, F. J. L. \& Gil, G. G. I. (2011). Globalization effects in family farms: a case of Mexican dairy production. In: Priot Pachura. The economic geographic of globalization. InTech, Rijeka, Croatia.

15. Kay, R. D. (1986). Administración agrícola y ganadera. Compañía editorial continental. Texas EUA. 432 p.

16. Leftwich, R. H. (1987). Sistema de precios y asignación de recursos. 9a ed. Nueva editorial Interamericana, México, D.F. 637 p.

17. Losada, H., Bennett, R., Cortés, J., Vieyra, J. \& Soriano, R. (2001). The Mexico City milk supply: Structure, function and sustainability. Agriculture and Human Values 18: 305-317.

18. Martínez, E. \& Salas, H. (2002). Globalización e integración regional en la producción y desarrollo tecnológico de la lechería mexicana. Miguel Ángel Porrúa Grupo editorial, Instituto de Investigaciones Sociales, UNAM, México. 291 p.

19. McDonald, J. H. (1999). The neoliberal project and governmentality in rural Mexico: Emergent farmer organization in the Michoacán highlands. Human Organization. 58 (3): 274-284.

20. Méndez, M. D., Tzintzun R. \& Val, D. (2000). Evaluación productiva, de efecto ambiental y de problemas relevantes en explotaciones lecheras de pequeña escala. Livestock Research for Rural Development 12 (1).

21. Nava-Tablada, M. E. \& Marroni, M. G. (2003). El impacto de la migración en la actividad agropecuaria de Petlalcingo, Puebla. Agrociencia 37 (6): 657-664. México

22. SAGARPA Secretaría de Agricultura, Ganadería, Desarrollo Rural, Pesca y Alimentación. (2004). Situación actual y perspectiva de la producción de leche de ganado bovino en México 1990-2000. Recuperado de: http//www.sagarpa.gob.mx.

23. Schwarzweller, H. K. \& Davidson, A.P. (2000). Dairy Industry Restructuring. Research in Rural Sociology and Development 8, New York. 411 p.

24. Valdespino O., J. (1993). Pérdidas por desecho prematuro de vacas en un hato lechero en México. Anim Zoot World Rev 5: 64-67.

25. Wiggins, S., Tzintzun, R., Ramírez, M., Ramírez, R., Ramírez, F. J., Ortiz, G., Piña, B., Aguilar, U., Espinoza, A., Pedraza, A. M., Rivera, G. \& Arriaga, C. (2001). Costos y retornos de la producción de leche en pequeña escala en la zona central de México. La lechería como empresa. Cuadernos de investigación. Cuarta Época 19, UAEM, Toluca, México. $61 \mathrm{p}$.

26. Zepeda, L. \& Jongsoog, K. (2006). Farm parents'views on their children's labor on family farms: A focus group study of Wisconsin dairy farmers. Agriculture and Human Values 23: 109-121. 